\title{
The Most Sensitive Arrangement of a Wheatstone Bridge with Special Reference to the Bolometer.
}

By C. D. Child and O. M. Stewart.

$\mathrm{T}$

HE usual theory of the Wheatstone bridge indicates that the most sensitive arrangement is secured when all of the resistances, including those of the galvanometer and battery circuits, are equal. ${ }^{1}$ However, this theory assumes that we have at our command an invariable electromotive force. In reality, one is usually limited not by his inability to secure a larger electromotive force, but by the thermal effects in the bridge circuits when too large a current is used. This is especially the case in using a bolometer. When the current in the bolometer exceeds a certain maximum, excessive heating effects occur, and irregular movements of the galvanometer are observed. This maximum current is fixed entirely by the properties of the bolometer strips.

It has been the usual practice to work out the theory of the bolometer on the assumption that the electromotive force is constant, and then, after connecting up the bolometer in the manner indicated by this theory, to proceed to vary the electromotive force until a certain maximum current in the bolometer strips is reached. As this theory assumed a constant electromotive force, while in reality the electromotive force is varied, one is not warranted in concluding that this is the most sensitive arrangement. In fact it seems probable that for this maximum current there might be some other arrangement which would be more sensitive.

Thus we see that in solving the equations of the bolometer connections for maximum sensibility, the current in the strips should be treated as a constant, and the electromotive force as a variable. From this standpoint Dr. H. F. Reid ${ }^{2}$ has shown that there are two solutions to the problem.

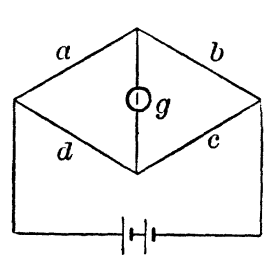

Fig. 1 .

(I) If, in Fig. I, $a$ and $b$ are the bolometer strips, the deflection of the galvanometer due to a small change in the resistance of the exposed strip is proportional to $\sqrt{\frac{1}{a+d}}$ and $d$ and $c$ should therefore be as small as possible. (2) If $a$ and $d$ are the bolometer strips, the deflection is proportional to $\sqrt{\frac{b}{a+b}}$, and, therefore, $b$ and $c$ should be as large as possible. As it has been found necessary to make the bolometer strips as nearly equal as possible, this equality was assumed in the derivation of the preceding results.

${ }^{1}$ Gray's Absolute Measurements, Vol. I., p. 331, and elsewhere.

${ }^{2}$ American Journal of Science, I35, p. 160. 
For the first case, however, Dr. Reid concludes that if the resistances $d$ and $c$ are made small, the current through them would be large, and the heating effects would cause much trouble. And, therefore, as the gain in diminishing $d$ and $c$ is not large, the best working results will be obtained when all the resistances are made equal. In the second case, when $a$ and $d$ are the bolometer strips, he says that if the resistances $b$ and $c$ are made large, heating effects would again follow. This, as before, is to be avoided, and the resistances should again be made equal. This last conclusion we regard as incorrect. There is a difference between the effect of heat developed in a small resistance by a large current, and that developed in a large resistance by a small current. In the first case the heat is concentrated in a short wire, while in the second case it may be distributed over a long one.

Our attention was called to this matter while one of the writers was engaged on the work described elsewhere in this number of the PHysical Review, p. 433. In that work one of the bolometers used was first connected in the traditional way. Each of the arms, including the galvanometer, had a resistance of about one ohm. This arrangement was afterwards changed, and the connections made as in Fig. I, where $a$ and $d$ represent the bolometer arms and $b$ and $c$ the other two arms, which were about 26 ohms each. The theory indicates that this change should produce a gain of about 30 per cent in the sensitiveness of the bolometer. The actual increase observed was in accordance with the predictions of this theory, and no increase in the unsteadiness of the galvanometer could be detected.

In order to verify the theory before reconstructing the bolometer connections, the following simple experiment was performed. The usual connections on a Wheatstone bridge were changed and others made as

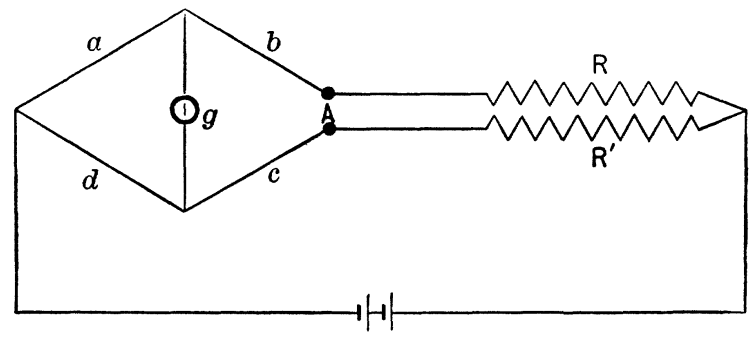

Fig. 2.

shown in Fig. 2. At $A$ were two mercury cups which could be connected at will. The resistances $R$ and $R^{\prime}$ were equal and were about roo ohms each. The resistance in each of the arms $a, b, c, d$ was one ohm. This arrangement fulfills the necessary condition of the theory, viz., that the 
current in the arms $a$ and $d$, which correspond to the bolometer strips, is constant. With connection made at $A$ the usual arrangement of the bolometer connections is given, but when not made at that point we have the second case of Reid's theory. The resistances were carefully balanced, both when the connection at $A$ was made and when it was broken. Then a slight increase was given to the resistance $d$, and the deflections of the galvanometer noted when connection was made at $A$ and when it was broken. In accordance with the theory the deflection was greater in the latter case than in the former. In fact there seems to be no doubt but that a gain of about 30 per cent in the sensitiveness of a bolometer is secured by changing the resistance from the form commonly used to one where the resistance of the arms not used for bolometer strips is large and the corresponding increase made in the electromotive force.

Theory indicates that the galvanometer should have a resistance $\frac{2 l R}{\mathrm{I}+l}$ where $R$ is the resistance of one of the bolometer strips and $l$ the ratio between this resistance and that of one of the arms not a bolometer strip. Often it is not practicable to change the resistance of the galvanometer. But for this case also, theory shows that the arrangement of resistances suggested in this article is preferable.

Physical Laboratory, Cornell University.

\section{Demonstration of the Doppler Effect.}

By R. W. Wood.

T treating of the Doppler effect in the class room, it is usual to cite the example of the change in the pitch of a locomotive whistle in rapid motion. As a matter of fact scarcely one person in ten has any distinct recollection of ever having noticed the phenomenon, unless his attention has been directed to it, and it may be of interest to teachers to know that it can be shown by means of a very simple and inexpensive piece of apparatus.

A small pitch pipe such as is used by musicians is securely fastened to the end of a tapering stick six or seven feet long, such as a blackboard pointer. This can be blown with a piece of rubber tubing fastened along the rod, and by waving the device rapidly, it is possible to give the sounding pipe a to-and-fro velocity of twenty or thirty feet a second, which is sufficient to cause a marked rise and fall in the pitch. By waving the stick first in the direction of the "line-of-sight," and then at right angles to it, it can be shown that the effect occurs only in the former case. A bamboo fish pole with the partitions bored out, the pipe fastened into the tip by means of sealing wax, and a short piece of rubber hose inserted near the breach, would make a less clumsy piece of apparatus. 